\title{
IMPROVING ABILITY AND LANE DETECTION OF SELF-DIRECTED-CAR
}

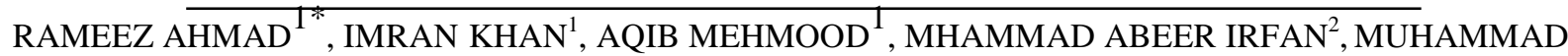 \\ ISMAIL $^{2}$, SAIMA NAEEM $^{1}$, EJAZULLAH $^{1}$, SYED HAIDER HASSAN $^{1}$, FASEEULLAH $^{3}$ \\ ${ }^{1}$ Department of Computing and Technology, Abasyn University, Peshawar, Pakistan \\ ${ }^{2}$ Department of Electrical Engineering, UET Peshawar, Pakistan \\ ${ }^{3}$ Department of Computing, UniversitiTeknologi Malaysia \\ Email: *ramizahmad@gmail.com
}

Revised November, 2015

\begin{abstract}
Driverless vehicles are on the move to announcement by Google, which drove more than 500,000 miles on its original model vehicles and further key automakers specify the prospective enlargement in this region with the capability to convert the transportation infrastructure, enlarge access and convey settlement to variety of user. A few users address the anticipated unfinished convenience of self directed cars by 2020 with accessibility to the community by 2040. Certain trust that self-directed car make necessary to renovate the modern transportation that fundamentally removing coincidences and cleaning up the road environment. This study understands the effects that self-driving car or robotic vehicle travel demands and ride scheme is likely to have, without the typical obstacles that allows detection of vision based hardware and software construction of SDC (self-directed car) technology and Gold (Generic Obstacle Lane Detection) to a knowledge-based system to expect the potential and consider the shape, color, balance in organized environment with colored lane patterns which is implemented by a particle filter. The algorithm is implemented and testing were approved on roads and the consequences show the strength of the algorithm to the problem natural in road location.
\end{abstract}

Keywords: Self-directed Car; Generic Obstacle Lane Detection; GPS; LIDAR

1. Introduction. Today we observe the traffic safety systems which are gradually increasing to reduce the danger of accidents around the world. There is a huge number of vision based systems for horizontal and longitudinal vehicle control, crash escaping and lane disappearance, which can play an important role in the organization. Intelligent traffic monitoring system is a growing need to develop during the last decade around the world. The car show trajectories object segmentation, tracking, partial and complete blockage can be obtained by screening performed. An obstacle detection method intellectual and overlapped objects in a number of areas are used for the account of vehicle changes lane keeping, lane geometry but stable video sequences can be estimated. Nonmetric distances to the various categories of functions, such as entry, exit, primary, or secondary system. Here we present a method that is independent of the camera view and it is in need of any special pre-calibration. Lane width supplies the words, even though the picture is not consistent across all pixel coordinates in a rough way "average" estimate lane width is required. Ability to eliminate the need for camera calibration corrections this process traffic standard without the burden of performing to allow the use of installations, as is particularly beneficial [1]. In the case of multiple road lane detection, the 
purpose of digital photo on center line of each lane is to find. This could possibly be in trees, bushes, humans, and others, as well as to meet the region of trademarks; other features can be used to differentiate the lane. Lane detection is tricky to analyzing the tracking and determines the lane markings which are not visible in the print quality sometimes and variations in environmental situations. The geometry of the mark as a bias element which cannot be used. Additional, the splitting, merging and detection can be bad interfering roadside objects or shadows. In this paper, we are able to manage with the difficulties that a novel detection algorithm planned operation. As long as the geometry of the lane patterns, speed, fusion, road marking materials to be realistic print quality of the road and stop the construction container, environmental variation and shadows can be tolerated in the system. The lane markings which hurdle by curbs, roadside objects and other features include expressive or meaningless objects in the image, the edge of the car on the side with a set of features based method is engaged. If the sides are weak and estimated by the resulting set of traditional lines, location and length of the coordinate transform gather by 3-D for three-dimensional and 2-D with the help of twodimensional. This approach describes the basis for the lane markings and restriction along the lines are long and is approximately the 3-D space are parallel. After another brief and isolated edge lines can be discarded in a different class, these lines will cluster together with Lane and stop edge lines, middle lines of the lane can be determined. As follows: Overall, this algorithm has qualities. First, this strategy works well under the terms of diagram surveillance. Second, clustering using the means to offer a robust approach. The center line is to determine just need of a picture frame as the third, the algorithm is effective. Fourth, it simultaneously computes the lane information. The fifth, is the center lines are precise enough for the intended application. The large amount of research work is reported effectively, especially for conditions like highway lane detection, tracking problem is solved in the literature. However, the shortcoming of urban road is to be used in the scenario. Usually several numbers of different obstacles being such as streets and lane markings detection to be able to track directly using [3] or only slightly curved lane Marking Tracking [4] [5].

2. Research Methodology. In this section we track the detection of lane marking which providing the functionality present a possible approach. After tracking the steps as we already have examples of the use of the information obtained. This section is meant to be a time step that notes tracking algorithm is summarized in Figure 1. In step 1, the filter is initialized as explained in lane detection. For this complicated task we require several particles to pick up all markings in following time steps, we know where to look for markings; therefore we place the particles only in the places of interest. That's why, the number of desired particles is lesser, which results in lower computational effort.

The algorithm shows a pseudo code program of a standard particle filter and include the parameter setting and main loops. The parameter setting contains set $\mathrm{P}$ of $\mathrm{N}$ particle condition and set $\mathrm{E}$ of estimated values of all particles that changes with time. The main loop have such steps as prediction, measurement and resample of particle condition, amongst the resample consists of the weight calculation of each particle condition and the position of new particle in re-sampling based on the weight value[17].

A pseudo-code for $\mathbf{P F}$ algorithm.

Procedure ParticleFilter()

Var

$\mathbf{P}[1 \ldots N]$ : Particle set;

$\mathbf{E}[1 \ldots N]$ : Estimate set along time;

$t$ : time;

begin

$t=0$

Initialize $(\mathbf{P})$;

while $(t<T)$ do begin

$\operatorname{Prediction}(\mathbf{P})$;

Estimate(P, E[t]);

Resample(P, E[t]);

$t=t+1$;

end

end 
The most important behavior in the form of very strong curves which occlude the mark that correspond to the absence of signs in the presence of vehicles. Figure 1 shows such a situation: as a Bookmarking occluding vehicle lane marking of the border is detected incorrectly. The visible part of the marking is correctly detected while still misdetection when no marking is detectable, where parts of the image is affected or absent. Figure 2. Results obtained by this approach and developed the first fully deterministic algorithm [6] obtained by a comparison between the show.

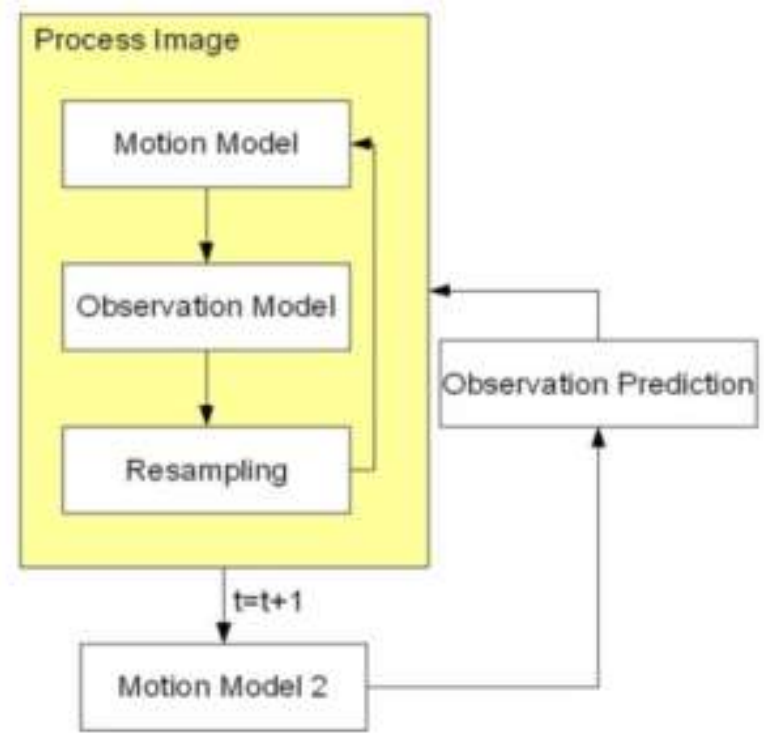

Figure-1: The Tracking Algorithm

Figure 2.a. As shown in the figure, straight-line is particular, deterministic approach and the most critical situation that may misdetection of marking in the presence of obstacles occluding the opposite lane markings, new stochastic approach in all situations in evidence of fast execution time but it is also positioned differently with vehicles or obstacles to a better precision and robustness. On the other side, the ant tracks are less flat than those calculated deterministically. Anyway, utilizing higher order interruption functions is presently under operation with better lane tracking system. Theme ant's liberate front frame [7] According to the results can be transferred to the necessary region.

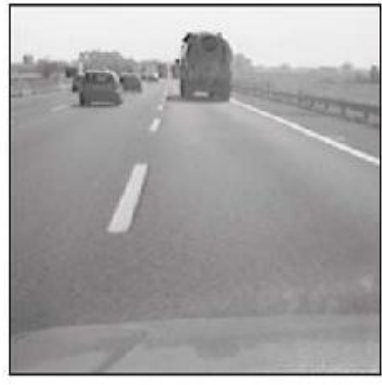

(a)

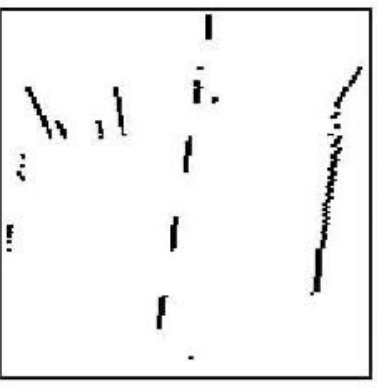

(b)

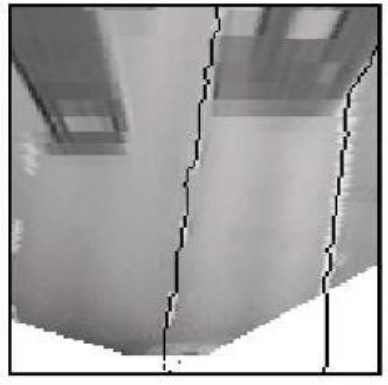

(c)

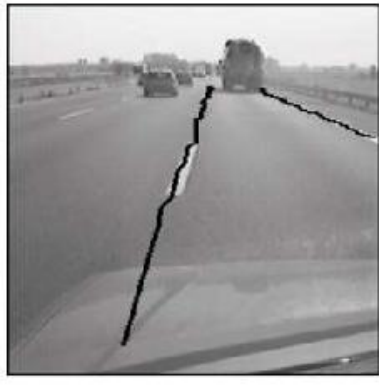

(d)

Figure-2: Example of serious state (blocking weakness)

(a) Unspoiled image, (b) Binaries, (c) Result covered onto the image acquired by the abstraction of the viewpoint effect (d) Result covered onto unspoiled image. 


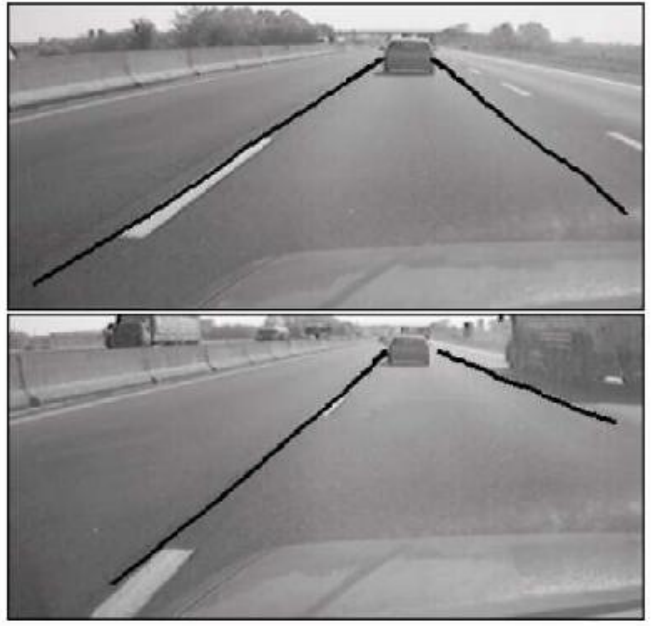

(a)

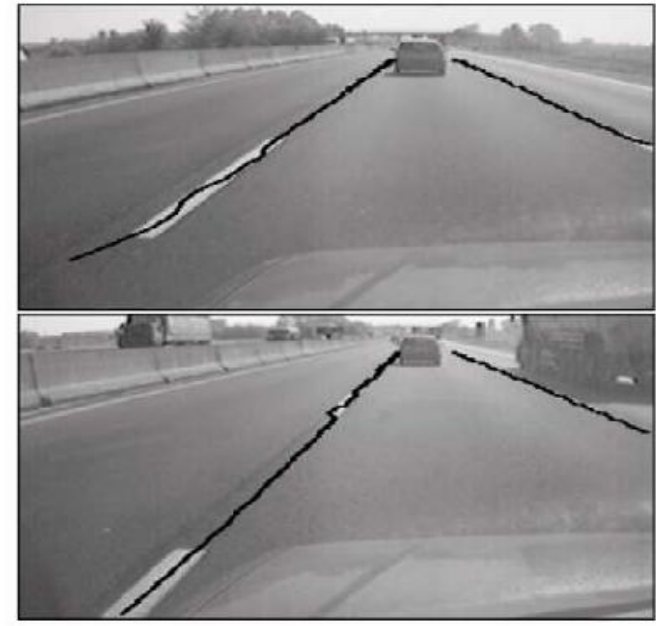

(b)

Figure-3: Different Methods evaluation.

(a) Consequences of the deterministic method (b) Results of the stochastic method

On the other hand, model-based technology trade represents only uses a few limitations. Assuming either dead straight line shapes $[10,11,12$, and 15] or parabolic curve $[8,9,13$, and 14] can be produced by detection of processing transactions Model calculations are contacted as processing parameters. In this way, the model-based technology in comparison with feature based technology is additional healthy against noise and lost data of Lane models, the possibility function $[8,9,10,11$, and 15] is to estimate the parameters, Hough's [2, 12] changed, and chi-square fitting [13, 14], applied to the detection of lane have been. Most Lane models only focus on the road are of some ways, however, such road [16] in the lack of random shape modeling.

\section{Problem Addressed.}

Self-directed car safely vehicle is driven over 700,000 miles. The car is tested on road but still cost increases. Additional vehicle equipment, services and maintenance and possibly require additional road infrastructure. Several time lane are lighten or not able to be seen, eg. 2 lanes, 3 lanes, and 4 lanes road in various developed and undeveloped states. Such aspects are becoming a difficulty to identify the lanes for Lane Departure Warning Systems (LDWS). Especially, when more lanes are present on road, so the detection algorithm may identify lanes by screening direction of camera inside a car.

3.1. Additional risks. The failure of such systems as the new threats, introduction to be safe under certain conditions and additional risks (offsetting behavior) make vehicle to travel and the growing external costs of road can encourage users traveling convenience and affordability, increasing autonomous vehicles parking, crashes and increase the external costs of pollution can bring additional vehicle travel. Social equity concerns. The convenience and other ways to protect the leads, for example, may have unfair effects.

3.2. Reduced Employment and business activity. Jobs for drivers should be rejected and the accident rate will be decrease due to limited vehicles and there may be less demand for repair. The technical solutions that focus on pedestrian and transit improvements of pricing improvements and other demand management strategies as traditional but effective implementation of transportation projects discourage communities could be a sign detections on the boards, the same meaning signs will indicating different complexity can cause the car to go off course.

3.3. Road construction. Lane architecture may differ from the ordinary roads. Positioning system GPS issues: self-directed car trust heavily on GPS satellites. A signal can be blocked and will affect the functionality of self-directed cars by horns, police and ambulance sirens or disturbing sound.

3.4. Age Range. The risk of life concerns "self-directed car can't be drive by someone". Crash accident, the driver or the machine is responsible for some reason when the accident took place. Major 
companies business can be affected such as taxi or Transport Company, buses and airline companies may be affected. hand signals.

3.5. Hand signals. Traffic police directing the traffic difficulty in understanding the manual or

3.6. Front view camera problems. Optical image sensor windshield and wiper may disturb the process.

\section{Proposed Method.}

The projected technique is to use an opposite perspective mapped image (IPM image) to run a particle set from the bottom to the top and observe the attendance of lane markings on each line. Additionally, to make sure that the filter is able to path various lines and to store each projected line as a track. This way produce an accurate data association, e.g. we associate each apparent piece of lane marking to one track, which signifies the marking of one lane which identifying two ways to implement this step. The first option is to recall the edge strength and color of the estimates, information which can be get from the top particle or an average including samples around the top particle throughout the detection phase. Then consider the ambiguity of a rough calculation, which however would suggest a damage of information. It is sensible to apply a number of observation prototypes to gain extra strength for the rough calculation. Presently we are using an edge image, which also encrypts the strength of the edges. The model adopts the stronger an edge is, the more possible it is to be part of a lane marking. This supposition is reasonable, because lane marking are normally structure on the road which are designed to be outstanding. Thus, these features should always be very changed from the surroundings. So the model can be defined as

$$
\omega e d g e(t)=\mu(y t \mid x t)=\frac{1}{\theta \sqrt{2 \emptyset}} \exp -\frac{1}{2} \frac{y t-\mu}{\mu}
$$

Where yt denotes the observation, in this case the edge strength at a particles position xt at time t. The edge strength is defined to be between 0.0 and 1.0. $\mu$ is the expected edge strength and is set to 1.0.

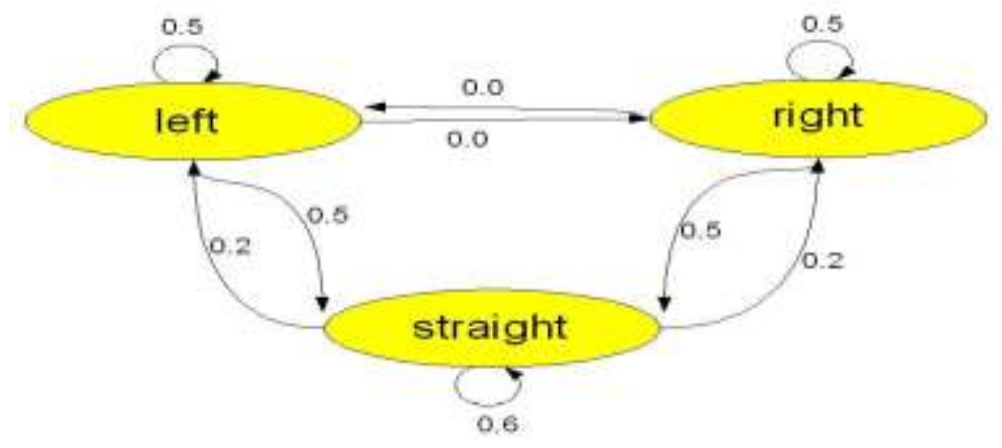

Figure-4: A simplified Markov model for lane marking detection.

Moreover, we use previous information about the color of the lane markings, e.g. whether these are white or Yellow and can therefore use the distance to this color as excellence measure, where the original image can be used. Again, this is a reasonable assumption because the lane markings color is different to that of the background (usually high contrast). The definition of this model, called $\omega$ color looks similar to 4 . Only yt- $\mu$ denotes the difference to the target color. The weight of the sample is then calculated wedge wcolor.

Self-directed car components:

- LIDAR

- Optical Image Sensor

- Radar

- GPS

- Wheel speed sensor / position estimator

- Processors 
4.1. LiDAR. HDL-64E LiDAR sensor. It continuously measures the surrounding environment that consists of 64 fixed-mounted lasers. Each laser mechanically the unit spins around while reading, it is mounted on a specific vertical angle. It created the production rate of 1.3 per million points.

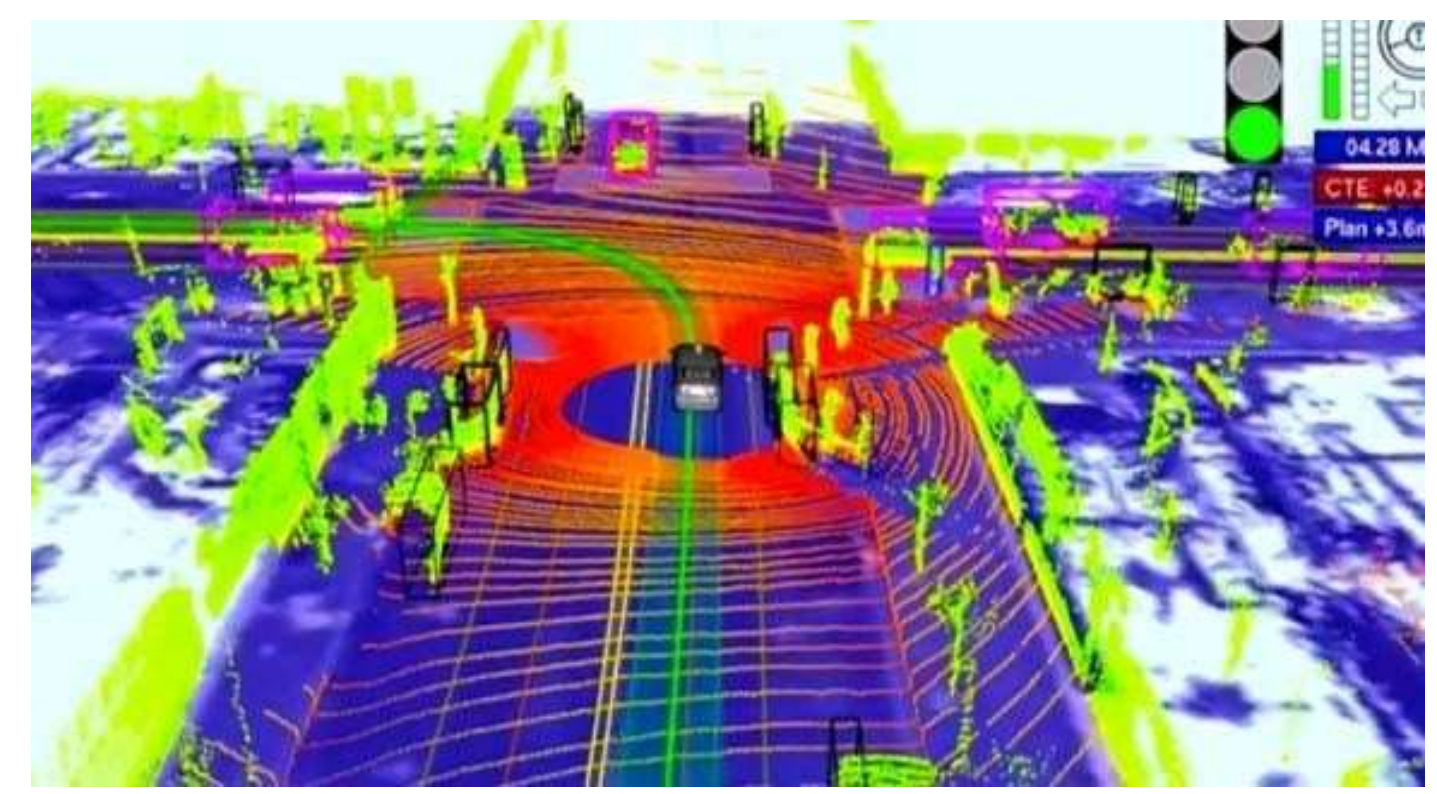

Figure-5: LIDAR Detection

4.2. Optical Image Sensors. A camera mounted near the rear-view mirror detects traffic lights and helps the car's onboard computers recognize moving obstacles like pedestrians and bicyclists. The basic purpose of an optical image sensor is to convert light into electrical signals. On cars of these sensors are used in several systems, including recorders accident warning systems and lane departure detection systems signs, parking assistance and avoid collisions. Optical sensors are usually based on one of two technologies CCD (Charge Coupled Device) or CMOS (Complementary Metal Oxide Semiconductor). Both sensors consist of an array of picture elements (pixels) which produce a current proportional to the intensity of illumination

4.3. CCD (Charge Coupled Device). CCD sensor elements, mainly the amount of light that strike the sensor directly proportional to the accumulated charge is a small capacitor. An array of sensors after an image has been exposed and charge a capacitor moved from its neighbor, the last capacitor in the array produces a voltage proportional to the charge amplifier is reduced in charge. This analog voltage level corresponding pixel location represents the brightness of the image.

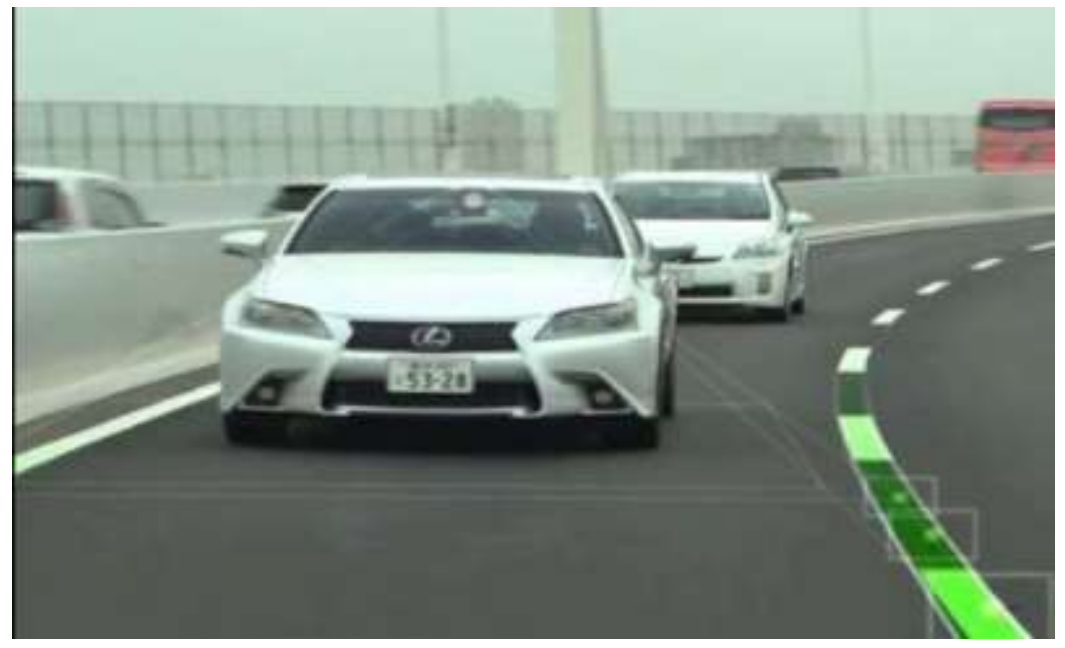

Figure-6: Optical image processing 
How will the car sense the traffic signals, a sensor "acidometer" is utilized to detect the intensity of radiation. Light of different colors will produce different intensity of radiation which will be detected by the sensor. If the detected intensity is of red color or yellow color. The controller will send a command to stop the transportation. The command will be followed by robot (to convert the computer command into mechanical input).

4.4. RADAR. Some cars and trucks are in front of a conveyance and the distance between any transportation and particularly object detection sensors are equipped with the progress. This sensor Adapt cruise control and collision avoidance systems. Most current progress $76.5 \mathrm{GHz}$ radar sensor utilized, but other frequencies (e.g.

$24 \mathrm{GHz}, 35 \mathrm{GHz}$ and $79 \mathrm{GHz}$ ) are with all in utilization. Some systems are utilizing radar sensors and infrared sensors. Utilizing radar to measure the distance are two rudimental ways. The first term is a function of the speed of light or in the time stay can be associated to the distance of the object reflecting the delay associated with the reaction of the imitated signal transmission method as directed and measures the transmission in receiving waves. The second method use directly frequency modulated continuous wave propagation method (FMCW) method is range of vision as directed to convey to modulate send and receive frequencies, frequency difference straight away as the relative speed of the object can be familiar to determine. Radar signals use dynamic electromagnetic radiation (e.g. metal objects) represents item that are strongly proficient while detecting. It works at a wavelength on the order of a few millimeters, due to several $\mathrm{cm}$ or more astronomically massive automotive radar system to detect objects that are very good. Withal minute relative to the wavelength (e.g. dihydrogen monoxide drop in fog (i.e. ignoring objects) are proficiently at looking through other self-propelled systems operating radar distance sensors include collision avoidance systems, blind spot detection and automatic car park system.

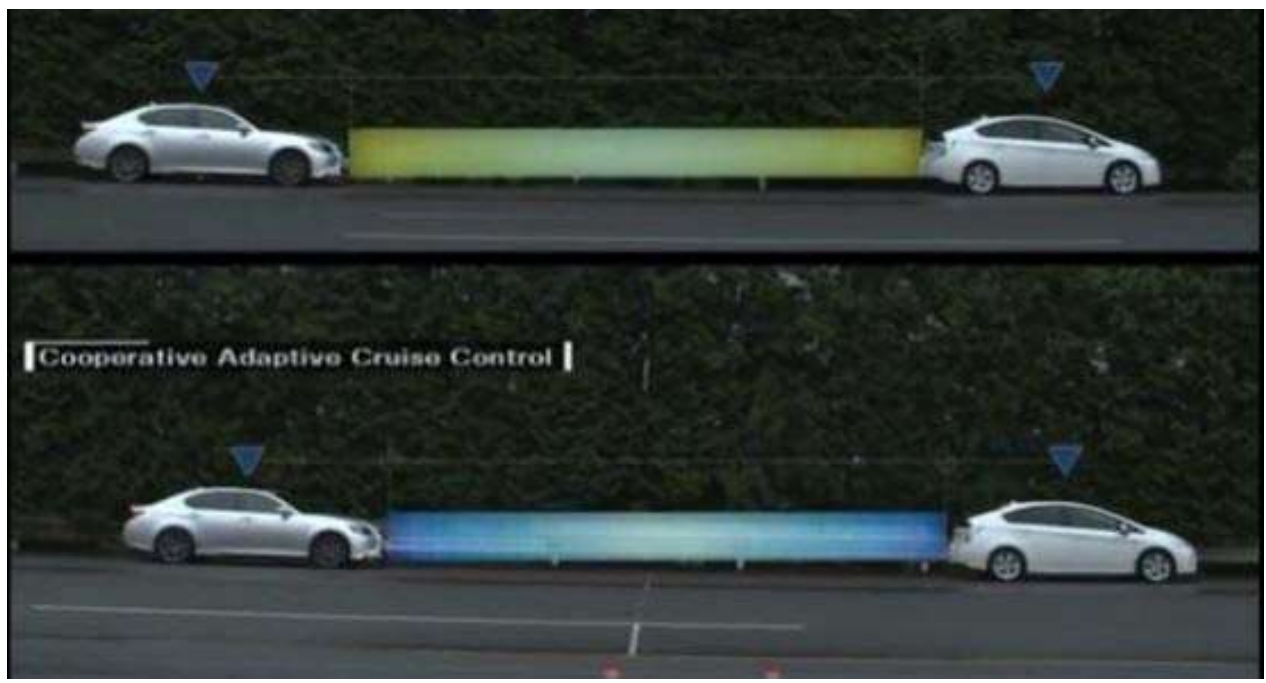

Figure-7: Radar processing

4.5. Global Positioning System (GPS). The satellite receives GPS satellite data in the form of high frequency signals of low power radio. The radio signals travel at the speed of light and the GPS receiver measures the distance between itself and the satellite time to find a radio signal to travel from the satellite to the own. However the satellite and the receiver to be able to measure the required precise clock. The satellites have an atomic clock; most commercial receivers utilize an ordinary quartz clock. The receiver measures the input signals of 4 or more satellites information than required just to set the position. Regular information is utilized to update the quartz watch receiver to maximize the correctness of their position calculations.

4.6. Sensors wheel speed. Wheel speed sensors calculated the speed and direction of rotation of the road wheel. The sensor has anti-lock brakes and electronic stability control systems including a variety of cars, a large number of inputs. Wheel speed sensors usually consist a toothed ring pickup (optical encoding) and axis (optical sensors) is magnetic sensors. Teeth or the speed at which the sensor is passing marks. Speed sensors are individual wheels do not need to know the speed at which vehicles can monitor wheel drive shaft or crankshaft rotation. Otherwise, monitoring each wheel rotation axis motor. Dual types of magnetic sensors: variable reluctance and Hall Effect. Both types of sensors as it rotates under a steel gear teeth out. Toothed comes in close closeness to a variable reluctance sensor as the change in inductance of a coil of wire detection. 
Hall Effect sensor due to an applied magnetic field in the power of a chunk of semiconductor measuring changes in resistance.

\section{Conclusion.}

In this work we discussed the most difficult task of lane marking detection as well as the task of lane marking, tracking, where we take the benefits and possible obstacles from the use of previous information. Using previous knowledge enables us to gain more robustness and at the same time to decrease the number of samples, as we already know where to place the sample at the beginning of a time step. As opposed to many previously presented algorithms we avoid strong theory about the geometry of a lane, which permit the filter to operate even in a difficult location where markings are obstructed or otherwise absent. Furthermore, even early implementations run in real-time, i.e. operate at least with the speed of our camera. Experimental results have established that the three optimization techniques function strongly in sensing the road and lane boundaries. Resolving the problematic result approaching for a self-directed-car will make it more efficient on roads.

\section{Future work.}

The further study is to improvement the intelligence of the sensors to detect when there is no mark or absent lane markings on the road. By improving the additional function of the intelligence of sensors will make the autonomous car more efficient.

\section{REFERENCES}

[1] Melo, J., Naftel, A., Bernardino, A., \& Santos, V. J. (2006). Detection and classification of highway lanes using vehicle motion trajectories. IEEE Transactions on Intelligent Transportation System, vol. 7, NO. 2.

[2] Lai, A.H.S., Yung. N.H.C (2000). Lane detection by orientation and length discrimination. IEEE Transactions on Systems, Man, and Cybernetics.

[3] Wang, R., Xu, Y., Libin, Y., \& Zhao. (2002). A vision based road edge detection algorithm. IEEE Intelligent Vehicle Symposium.

[4] Kluge, K. (1994). Extracting road curvature and orientation from image edge points without perceptual grouping into features. Proceedings of the Intelligent Vehicles Symposium.

[5] Sehestedt, S., Kodagoda, S., \& Alempijevic, A. (2007). Efficient lane detection and tracking in urban environments. The Arc Centre of Excellence for Autonomous Systems, EMCR, Australia.

[6] Bertozzi, M., Broggi, A., \& Fascioli, A. (2000). Visual perception and learning in road environments. 6th International Conference on Intelligent Autonomous Systems, IAS-6, Venice, Italy.

[7] Bertozzi, M., Broggi, A., Fascioli, A., \& Tibaldi A. (2002). An evolutionary approach to lane markings detection in road environment. I-43100, Parma, Italy.

[8] Kluge, K., Lakshmanan, S. (1995). A deformable template approach to lane detection. I. Masaky (Ed.), Proceedings IEEE Intelligent Vehicle, 95, Detroit.

[9] Lakshmanan, S., Kluge, K. (1995). Lane detection for automotive sensor. ICASSP 2955-2958.

[10] Grimmer, D., Lakshmanan, G. (1996). A deformable template approach to detecting straight edges in radar images. IEEE Transactions on Pattern Analysis and Machine Intelligence, (18), 438-443.

[11] Kaliyaperumal, K., Lakshmanan, S., \& Kluge, K. (2001). An algorithm for detecting roads and obstacles in radar images. IEEE Transactions on Vehicular Technology, 50 (1), 170-182.

[12] Kang, D. J., Choi, J. W., \& Kweon, I. S. (1996). Finding and tracking road lanes using line-snakes. Proceedings of Conference on Intelligent Vehicle, pp. 189-194, Japan.

[13] Kaske, A., Wolf, D., \& Husson, R. (1997). Lane boundary detection using statistical criteria. International Conference on Quality by Artificial Vision, QCAV9 28-30. France.

[14] Kaske, A., Husson, R., \& Wolf, D. (1995). Chi-square fitting of deformable templates for lane boundary detection. IAR Annual Meeting 95, Grenoble, France,.

[15] Liou, S. P., Jain, R.C. (1987). Road following using vanishing points. Computer Vision, Graphics, and Image Processing, 39.

[16] Wanga, Y., KhwangTeoh., E., \& Shen, D. (2004). Lane detection and tracking using B-snake, Image and Vision Computing, 269-280.

[17] Cheng, W. C. (2012). PSO algorithm particle filters for improving the performance of lane detection and tracking systems in difficult roads. Sensors, 12(12), 17168-17185. 